Ciencia y Educación, Vol. 5, No. 2, mayo-agosto, 2021

ISSN (impreso): 2613-8794・ ISSN (en línea): 2613-8808

DOI: https://doi.org/10.22206/cyed.2021.v5i2.pp81-92

\title{
Revisión teórica del concepto agencia. Implicancias educativas para comprender el compromiso académico
}

\author{
Theoretical review of the agency concept. Educational implications \\ for understanding the academic engagement
}

Daiana Yamila Rigo ${ }^{a}$ ORCID: 0000-0001-6274-5815

Rosana Beatriz Squillarib ORCID: 0000-0001-6274-5815

Mariela Rosana Caraballoc ORCID: 0000-0001-6371-2900

Romina Rovere ${ }^{\mathrm{d}}$ ORCID: 0000-0002-5843-6670

Recibido: 22/06/2020 • Aprobado: 12/08/2020

Cómo citar: Rigo, D. Y., Squillari, R. B., Caraballo, M. R., \& Rovere, R. (2021). Revisión teórica del concepto agencia. Implicancias educativas para comprender el compromiso académico. Ciencia y Educación, 5(2), 81-92. https://doi.org/10.22206/cyed.2021. v5i2.pp81-92

Resumen

El trabajo presenta una revisión bibliográfica del concepto agencia, contemplando el estado actual de la investigación y la discusión científica con respecto a los aportes teóricos más importantes, tomando como referencia central la Teoría cognitiva social. Se parte de la delimitación conceptual de la agencia personal, en sentido general, y, en particular, con relación al constructo compromiso académico, como la dimensión proactiva que tiene un fuerte impacto para pensar las prácticas educativas de nivel superior. Seguidamente, se describen aportes teóricos convergentes, retomando contribuciones desde la teoría histórico-cultural de la actividad, los planos de la actividad sociocultural y la influencia de la teoría para repensar el campo educativo. Finalmente, se avanza hacia la

\begin{abstract}
The paper presents a bibliographic review of the agency concept, contemplating the current state of scientific research and discussion regarding the most important theoretical contributions, taking social cognitive theory as a central reference. It starts from the conceptual delimitation of personal agency, in a general and particular sense in relation to the academic engagement construct, as the proactive dimension that has a strong impact to think about higher level educational practices. Next, describe convergent theoretical contributions, taking up contributions from the historical-cultural theory of activity, the levels of sociocultural activity and the influence of theory to rethink the educational field. Finally, progress is made towards the definition of criteria to be considered in
\end{abstract}

\footnotetext{
a Instituto de Investigaciones Sociales, Territoriales y Educativas, Consejo Nacional de Investigaciones Científicas y Técnicas, Universidad Nacional de Río Cuarto, Argentina. Correo-e: dyrigo@hum.unrc.edu.ar

b Universidad Nacional de Río Cuarto, Argentina. Correo-e: rsquillari@hum.unrc.edu.ar

c Universidad de Mendoza, Argentina. Correo-e: mariela.caraballo@um.edu.ar

d Universidad Nacional de Río Cuarto, Argentina. Correo-e: rominarovere@hum.unrc.edu.ar
} 
delimitación de criterios a contemplar en una educación que busque promover el compromiso agente a través del aprendizaje expansivo, donde la agencia se piensa como múltiple, distribuida y en red.

Palabrasclave: aprendizaje expansivo, agencia, compromiso, educación superior, contexto sociocultural.

\section{Construcción conceptual}

Atendiendo a los lineamientos de Kuckartz y Rädiker (2019), se realiza una revisión sistemática para identificar, evaluar y sintetizar trabajos y aportes realizados por investigadores, académicos y profesionales sobre la agencia personal, con el objetivo de determinar el estado actual del concepto. La agencia tiene una larga trayectoria dentro del campo de investigación, en general, y, en particular, en el educativo. Cobra en la actualidad una nueva impronta tras ser incorporado como dimensión del compromiso académico. Esa incorporación reclama atender a una revisión de las contribuciones que a nivel teórico se tiene en la comunidad científica para pensar las prácticas educativas.

Entre los aportes cruciales, la revisión de la Teoría cognitiva social, desde Bandura, es elocuente para profundizar sobre la agencia personal. Si bien no es materia ahondar en la teoría en general, los desarrollos del autor con relación al concepto en cuestión merecen un tratamiento especial. Bandura (1989), en el siglo pasado, remarcaba el interés de estudiar los fenómenos autorreferenciales, no solo para otorgar valor y significado a las influencias externas, sino como determinantes próximos de la motivación y de la acción; exponiendo que la capacidad de controlar los propios procesos de pensamiento, motivación y acción son eminentemente humanos, a partir de los cuales el sujeto puede llevar a cabo cambios sobre sí mismo y sus situaciones.

En esa primera contribución y en las siguientes Bandura (1989, 2001, 2006) afirma que la mente de las personas no es solo reactiva, sino proactiva, an education that seeks to promote agent engagement through expansive learning, where the agency is thought of as multiple, distributed and in red.

Keywords: Expansive learning, agency, engagement, higher education, sociocultural context.

autorregulada, reflexiva y creativa; idea que subyace a la definición de agencia como la capacidad que poseen los sujetos para transcender el contexto inmediato y para dar forma a las circunstancias y los cursos de la vida. Ser agente supone la intencionalidad en las acciones llevadas a cabo por las personas, como contracara de un sujeto pasivo que reside en su contexto, sin influir, ni mediar en los procesos de autodesarrollo, autoadaptación y autorrenovación en tiempos cambiantes.

La agencia, así definida, está compuesta por cuatro propiedades: la intencionalidad, que incluye la realización de planes de acción para conseguir las metas propuestas, haciendo uso de la autoeficacia, expresando un compromiso proactivo para alcanzarlo; acciones en las cuales se ligan intenciones compartidas, donde se fusionan diversos auto-intereses; la previsión, que implica una extensión temporal de la agencia, debido a que incluye la fijación de metas y la anticipación de resultados probables de sus acciones para motivar los propios esfuerzos. Hace a la capacidad de transcender lo pautado, regular el presente y acceder a un futuro afín a los auto-intereses; la autorreactividad, que hace referencia a la autorregulación de los cursos de acción, atravesada por las metas propuestas, las características que poseen, tales como el nivel de desafío y la proximidad temporal; y, por último, la autorreflexión, que es la examinación del propio accionar. Es una actividad metacognitiva a partir de la cual las personas contrastan y juzgan sus planes y predicciones con los resultados de sus acciones y las de los otros. En este plano, las creencias de autoeficacia son importantes, en tanto influyen sobre el propio funcionamiento y sobre los eventos del contexto (Bandura, 2001; 2006). 
En la descripción de las propiedades, se visualiza que hablar de una agencia personal es una perspectiva mezquina, en tanto se puede reconocer una agencia representada y otra colectiva. En muchos entornos, las condiciones y prácticas sociales e instituciones que afectan la vida cotidiana o las prácticas educativas de las personas no están a su alcance. En esas circunstancias, buscan modificar los escenarios a través del ejercicio de la agencia representada. Asimismo, la agencia personal se puede pensar dentro de las influencias socio-estructurales, es decir, muchos de los objetivos que se buscan son alcanzables solo a través del esfuerzo compartido, o sea, del trabajo coordinado con otros (Bandura, 2006).

Al respecto, Wertsch (1998) puntualiza que los esfuerzos encaminados a pensar que la acción humana se centra meramente en el agente individual son restringidos. Retomando la péntada de Burke -acto, escena, agente, agencia y propósito-, a modo de responder al qué, dónde, quién, cómo y por qué, el autor analiza la acción humana, unida a los motivos, para explicar que el contexto no es algo sobre lo cual hay que actuar solamente, sino con lo cual hay que interactuar, donde, junto a los objetivos y los instrumentos, se asume una complejidad con profundas implicancias metodológicas para los estudios socioculturales. El análisis de cada acción queda supeditada a la infinidad de combinaciones de los 5 elementos.

El interjuego de los elementos que definen la péntada deriva en sentidos múltiples, en tanto, como lo expresa Sautu (2014), desde una perspectiva sociológica, la agencia humana es dinámica, en tanto socio-históricamente conformada a lo largo de las trayectorias de vida. Es decir, que tanto la agencia, como la estructura, se relacionan en el contexto de los procesos socioculturales y políticos-económicos (Ávila Reyes, Navarro y Tapia-Ladino, 2020). Estas estructuras aparecen en las situaciones, donde el comportamiento de los agentes se desarrolla junto a recursos materiales y simbólicos, que delimitan oportunidades y obstáculos.

Asimismo, los elementos centrales -intencionalidad, previsión, autorreactividad y autorreflexión- del agente permiten a las personas ser parte de su progreso, adaptación y transformación a través del tiempo. Sin embargo, no se puede omitir que las acciones de los agentes están contextualizadas y que las condiciones básicas estructurales pueden ser posibilitadoras o limitadoras de la agencia personal. En este marco, la agencia humana ha sido conceptualizada como interactiva. Bandura (1999) plantea que la mayor parte de las herramientas a través de las cuales la agencia se expresa derivan de las experiencias sociales. Es decir, la acción humana está socialmente situada y es producto de la interacción dinámica entre influencias personales y contextuales.

La interrelación entre los rasgos personales y contextuales es retomada en los desarrollos teóricos sobre el compromiso académico, como metaconstructo modificable, dinámico y situado, para definir sus dimensiones afectiva, conductual y cognitiva, y una cuarta más reciente, denominada agéntica (Rigo y Donolo, 2019). Reeve y Tseng (2011) comprenden que los estudiantes no solo reaccionan a actividades de aprendizaje proporcionadas por los docentes, sino también actúan sobre ellas, modificándolas y enriqueciéndose. Los estudiantes reaccionan prestando atención, disfrutando de la tarea o utilizando estrategias profundas, pero a la vez participan activamente en el proceso de aprendizaje para enriquecer la actividad, modificarla, permitirse mayor autonomía, tener un mayor acceso a los medios necesarios para mejorar la comprensión, a modo de contribuir constructivamente, desde la agencia personal, al flujo de la enseñanza que reciben.

El compromiso agente es definido por Reeve (2013) y Reeve y Shin (2020) como los actos intencionales, proactivos y constructivos que los estudiantes despliegan para mejorar sus oportunidades de aprender, enriqueciendo la experiencia educativa y sus aprendizajes. Los estudiantes contribuyen constructivamente a la dinámica de los procesos de enseñanza, mediante transacciones dialécticas entre alumno y profesor, que conducen a negociar un entorno de aprendizaje más motivador y de mayores apoyos. En esa negociación se piensa al aprendizaje como reconfiguración de agencia, 
(...) entendida como una transformación del repertorio de acciones intencionales que se espera que alguien pueda desplegar en el contexto de la práctica social o de sistemas de actividad específicos. Dicha reconfiguración es resultado de la participación misma de la persona en el contexto social y es coextensiva a la participación de la persona en nuevas posiciones, bien dentro de un sistema de actividad en el que la persona ya cuenta como participante, bien en nuevos sistemas de actividad. En tal sentido, es decir, en la medida en que el aprendizaje es coextensivo de la participación, la persona es al tiempo agente y paciente de su aprendizaje; vale decir, de su práctica social. (Larreamendy Joerns, 2011, p. 40)

Este aprendizaje no puede significarse como práctica social en sí mismo, en tanto refiere a los cambios que la colaboración de los agentes genera en y sobre la práctica social. Las personas, los alumnos y los docentes se mueven entre contextos diversos, siendo que la agencia se desarrolla entrelazada en comunidades de práctica que, con restricciones y posibilidades, definen lo que es posible en el contexto de una actividad, buscando nuevas posiciones y nuevos movimientos; acciones de los agentes que conllevan cambios en las formas de implicarse (Larreamendy Joerns, 2011).

De manera interconectada al aprendizaje como reconfiguración de la agencia, cobran crucial importancia los intereses presentes en los sistemas de actividad, las prácticas sociales o en los campos. Retomando a Bourdieu (1997), en la metáfora del 'juego' para analizar el illusio, o el interés de jugar el juego porque vale la pena jugarlo, se muestra la existencia de intereses diversos como campos existentes, posiciones ocupadas y trayectorias educativas recorridas; estar implicado y jugar el juego académico, y desde la posición de alumno, es estar interesado en participar de las actividades que se presentan como prácticas hacia mejores aprendizajes; una illusio que se conserva y se expresa, trasformando el campo, en tanto el contexto educativo habilita un espacio de juego dialéctico, in tándem, docentes y pares (Rigo, en prensa).

$\mathrm{Al}$ respecto, Bandura (2006), haciendo nexo entre la noción de illusio y agencia, argumenta que la agen- ciación humana implica justamente intencionalidad con los planes y las estrategias que podría demandar la acomodación de los intereses propios con los expresados por otras personas, como ser los docentes. Intención que Reeve y Jang (2006) definen como la decisión de participar en un comportamiento particular, equivalente a estar motivado para actuar. Esta puede estar originada en nosotros mismos, coaccionada o presionada por otro agente, determinado el grado de implicación de los sujetos. Por lo que, la interacción profesor-alumno es un factor esencial para experimentar la agencia en la clase (Montenegro, 2017). Al respecto, Zuckerman (2007) conceptualiza esas interrelaciones como supraindividuales, es decir, un lugar donde dos intenciones se encuentran y se enriquecen mutuamente. En otras palabras, apoyar las iniciativas de los estudiantes dirigidas hacia un objetivo específico significa construir una cooperación mutua activa entre los agentes educativos.

En síntesis, la agencia como constructo que forma parte del compromiso académico de los estudiantes, muestra ser una construcción social, mediada por las posibilidades y las limitaciones que el entorno educativo delimita en las interrelaciones que se configuran en el aula, en función de las herramientas culturalmente situadas. La agencia personal de los sujetos se expresa entrelazada a los comportamientos de los docentes (y pares) que apoyan o no esas decisiones proactivas e intencionales de participar en función de las metas personales y pedagógicas, moldeando el entorno próximo.

\section{Aportes teóricos diversos}

En este apartado avanzamos sobre los postulados y la evolución de la teoría de la actividad, desde un enfoque sociocultural, retomando los planteamientos centrales de Engeström y los específicos de Rogoff, para entender los modos de participación de los agentes en los sistemas de actividad. Finalmente, se introduce una síntesis del impacto que la teoría tiene para pensar en nuevos contextos educativos que promuevan el compromiso académico de los estudiantes. 
La teoría de la actividad tiene como finalidad comprender los sistemas de actividad a partir de los cuales las acciones de los agentes cobran sentido. Más precisamente, Engeström (2008) define que la teoría de la actividad está dirigida por objetos, es decir, preocupaciones, focos de atención, motivaciones y esfuerzos.

Los aportes de Engeström dan origen y nutren a la tercera generación de la teoría de la actividad, precedida por otras dos generaciones. La primera, sustentada en la idea de mediación cultural de Vygotsky
(1993), expresada en la triada sujeto, objeto y artefacto mediador; y, la segunda, construida sobre la noción de actividad de Leóntiev (1989), quien suma a la triada vigotskiana un cuarto elemento, representando a la comunidad.

La base de la tercera generación se encuentra en la idea de diálogo y la multiplicidad de perceptivas que interaccionan sobre un objeto compartido. En su formulación inicial, toma dos sistemas de actividad que interactúan para la construcción de objetos (Figura 1).

\section{Figura 1}

Sistemas de actividad en interacción como modelo minimo para pensar la tercera generación

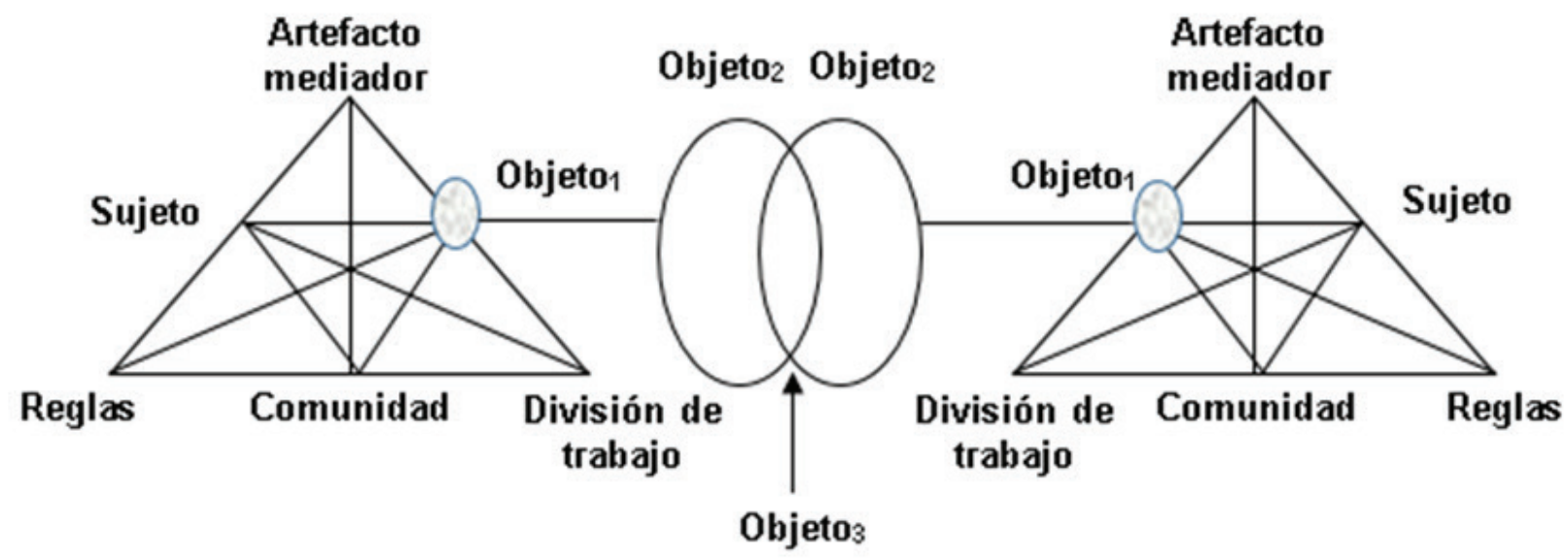

Nota. Adaptada de Engeström, 2001.

En la Figura 1, el objeto 1 se mueve de un estado inicial (por ejemplo, un estudiante que entra a clase) a un objeto 2 de importancia colectiva, construido por el sistema de actividad (por ejemplo, el estudiante como sujeto de aprendizaje); y un objeto 3, compartido o construido colectivamente (como la comprensión de la situación de enseñanza y el plan de actividades a desarrollar por el alumno para aprender). El objeto de la actividad está en constante movimiento y no puede reducirse a metas de corto plazo (Engeström, 2001).

Ineludiblemente, los sistemas de actividad, incluso en el campo educativo, entran en crisis. La naturaleza social de los procesos educativos, su comple- jidad interna e interconectada, así como su enorme capacidad de producción, hacen evidente, al menos en periodos de cambios intensos, que nadie domina la actividad educativa en su conjunto, conformando zonas grises, donde la acción intencionada de cualquier agente puede tener efectos no esperados (Engeström y Sannino, 2010). En tal sentido, Rigo y Donolo (2018) plantean que el docente ya no es el único que tiene el saber. Los estudiantes pueden acceder al mundo del Internet como una gran biblioteca que abre un abanico de recursos que invita a ser aprendices más autónomos en la tarea de aprender. 
El planteo de la interconexión lleva a pensar en la necesidad de un aprendizaje expansivo. Rigo y Guaroni (2020), recientemente, afín a la complejidad de los procesos educativos, exponen la idea de expandir el clima de la clase para generar rupturas con la idea de aprender alrededor de cuatro paredes que delimitan el espacio físico del aula. El potencial interactivo del internet abre un campo novedoso para la formación de actividades, con un enorme potencial expansivo; aprendizaje expansivo "en el que los individuos participan en la construcción y aplicación de un objeto y de un concepto nuevo, más amplio y complejo para su actividad" (Engeström y Sannino, 2010, p. 417). Es decir, a través de sus actividades las personas cambian constantemente y crean nuevos objetos. Los nuevos objetos a menudo no son producciones intencionales de una sola actividad, sino consecuencias de múltiples sistemas de actividades en los que participan los agentes, los cuales pueden ser caracterizados como objetos fugitivos. En la actualidad, lidiamos con 'objetos fuera de control', que nombrados y significados por los sujetos se vuelven un desafío y alientan a generar soluciones comprometidas y creativas a partir de los intercambios entre los participantes que actúan sobre el objeto ${ }^{a}$. Aunque, podría tratarse también de objetos innovadores, de emancipación, con potencial para generar nuevos desarrollos.

Muchos de los objetos fugitivos crean controversias, oposición y conflictos. Las implicancias del concepto para el campo educativo y del compromiso académico son interesantes para pensar la problemática de la desvinculación. La participación de los estudiantes en distintas actividades cambia y genera nuevos objetos, nuevas atenciones, motivaciones y significados, producto de las interacciones con pares y docentes en escenarios diversos, lo que habilita hablar de una construcción compartida de objetos. Rigo, Irusta, Bechero y Amaya (2020) plantean que, a lo largo del tiempo, los procesos de desvinculación van adoptando diversos motivos. En este marco, ¿podría la desvinculación desarrollar paulatinamente un protagonismo no advertido llegando a transformar las prácticas de manera casi viral para convertirse en un objeto fugitivo? Tanto por su carácter controversial como por su potencial para crear nuevas posibilidades puede ser tal.

En la actualidad, el conocimiento ya no es exclusivo de los docentes. El uso de wikis es un buen ejemplo de cómo se ofrecen oportunidades ilimitadas a los estudiantes de acceder al conocimiento (Rigo y Donolo, 2018). Los múltiples sistemas disponibles para aprender ponen en discusión y crisis los actuales contextos de enseñanza, los procesos de participación y atención de los alumnos; problemática que se extiende rápidamente y que pone a los sistemas educativos a pesar en posibles formas de innovar para atender y expandirse hacia nuevos contextos de producción de saberes y de interacciones antes no incluidos. Así, los procesos de implicación (y desvinculación) podían ser definidos como objetos fugitivos, en tanto adquieren nuevos significados que transcienden las fronteras del aula, habilitando nuevos escenarios, junto a nuevas herramientas culturales de aprendizaje.

Engeström (2001) formula que los ciclos expansivos inician con un cuestionamiento por parte de los agentes o grupos de sujetos de las prácticas coronadas, expandiéndose hasta conformar un movimiento colectivo o un nuevo sistema; allí se hacen visibles 5 principios que caracterizan a la teoría de la actividad: el trabajo en red, la multiplicidad de voces; la historicidad; las contradicciones y las transformaciones expansivas.

Contemplando los cinco principios es que se entiende al aprendizaje "como una construcción entramada en el sistema de actividad y vinculada a los diferentes modos de participación de los sujetos" (Arrúe y Elichiry, 2014, p. 66). Con respecto a los modos de participación, Rogoff $(1993 ; 1997)$ formula tres planos donde tienen lugar las actividades socioculturales: la apropiación participativa, la participación guiada y el aprendizaje. La participación guiada se refiere a los procesos interpersonales de colaboración recíproca entre los individuos, como parte de una

a. La actual pandemia COVID-19 es un objeto fuera del control, el cual depende fuertemente de las actividades y acciones de los agentes para encontrar soluciones entrelazadas entre países, potencias y culturas diversas. 
actividad culturalmente significativa; en esa actividad cara a cara los individuos se van transformando, emergiendo la apropiación de la participación -personal- o el modo en que los sujetos evolucionan a través de la colaboración en una u otra actividad, cuando piensan, re-piensan, recuerdan, planifican, como procesos activos que conducen a nuevos aprendizajes y responsabilizan para llevar a cabo una actividad semejante a futuro.

La transformación de la participación, vinculada al concepto de aprendizaje expansivo, se manifiesta principalmente como cambios en el objeto de la actividad colectiva, que conduce a una transformación cualitativa de todos los competentes del sistema de actividad. A la inversa, se pensaría desde una visión tradicional, donde el cambio se valora solo en el sujeto, en sus comportamientos y procesos cognitivos (Engeström, 2001).

Wertsch (1998) menciona que la introducción de nuevas herramientas culturales transforma la acción, es decir, genera una especie de desequilibrio en la organización sistémica de la acción mediada que conduce a cambios en otros elementos, tal como el agente y la misma acción mediada que podría ser incluso totalmente novedosa. Erausquin (2014) reflexiona sobre cómo los artefactos culturales delimitan formas particulares de apropiación e intercambios y aluden a las experiencias educativas que provienen del cruce de fronteras, retomando a Engeström (2008), para ejemplificar la importancia de crear puentes entre sistemas de actividad de diversos espacios socioculturales para expandir el aprendizaje encapsulado.

Lo planteado es elocuente para pensar la influencia de las nuevas y diversas herramientas culturales sobre los procesos de aprendizaje. Gutiérrez, Engeström y Sannino (2016) y Laitinen, Sannino y Engeström (2016) formulan que la agenda es audaz cuando se propone desde el campo de la investigación educativa contribuir a la expansión de la educación más allá de los entendimientos ya establecidos de enseñanza y de aprendizaje, remarcando dos características importantes. Primero, que aprender y que enseñar van más allá de las aulas y las escuelas, ingresando a una variedad de comunidades, actividades y movimientos sociales que son vistos como sitios potencialmente significativos de aprendizaje y de enseñanza. Segundo, las metodologías de investigación van más allá de la observación y las metodologías lineales como los ensayos controlados aleatorios, ingresando diseños participativos, tal como las intervenciones formativas, los experimentos de diseńo social y los diseños basados en la comunidad. Este abanico de posibilidades puede verse como una zona colectiva de desarrollo próximo ${ }^{\mathrm{b}}$.

En este marco, Yamazumi (2006; 2007; 2009) plantea la creación de nuevas agencias distribuidas, múltiples y en red, al interior de su teoría pedagógica emergente basada en el aprendizaje expansivo propuesto por Engeström, exponiendo la importancia de trascender los límites institucionales, a través de sistemas de aprendizaje fuera de la escuela o en contextos híbridos. Con el aprendizaje expansivo se busca la creación de nuevas prácticas pedagógicas, donde las personas involucradas en los procesos instructivos pueden diseñar y moldear el contexto educativo, ante la presencia de criterios asociados a la desvinculación académica, es decir, apatía, falta de interés, aburrimiento, como síntomas de la educación que se aleja de los motivos que comprometen (Rigo et al., 2020).

El enfoque de aprendizaje expansivo aprovecha los conflictos e insatisfacciones reales entre los profesores, estudiantes, padres y otras personas involucradas o afectadas por la educación, incitándolos a unirse a una metamorfosis concreta de la práctica actual. En otras palabras, de las contradicciones actuales, los participantes hacen un análisis conjunto para pensar en formas alternativas de mejorar la educación (Engeström, 1991).

b. Nuevamente, la actual pandemia nos mueve de la zona de confort hacia la inclusión de la educación virtual como modelo alternativo a las tradicionales arraigadas formas de aprender en contextos presenciales, la evolución hacia contextos híbridos, ya presentes, será casi materia obligatoria en el futuro. 
En síntesis, la teoría de la actividad histórico-cultural se presenta como un nuevo paradigma que analiza el aprendizaje como producto de las interacciones entre individuos y colaboraciones dentro de las comunidades. La introducción de tecnologías de la información y de la comunicación como el Internet abre un escenario de aprendizaje cada vez mayor para los estudiantes y los profesores, donde el aprendizaje ya no es algo que tiene lugar dentro de los límites de los libros de texto. En la mayoría de instituciones educativas de nivel superior, las actividades culturales, los problemas sociales y las posibilidades futuras forman una parte esencial de los planes de estudios. En consecuencia, es cada vez más importante que las universidades forjen alianzas con organizaciones comunitarias, expertos y otros escenarios relevantes que posibiliten relaciones recíprocas para crear y compartir conocimientos y prácticas donde profesores y estudiantes se involucren con temas y problemas interesantes por investigar e intervenir fuera del aula (Yamazumi, 2007).

Bandura (2001), a principios de siglo, advertía sobre cómo los cambios a nivel informático, social y tecnológico impactarían en la autoeficacia para el autodesarrollo y la autorrenovación. En el pasado, y en muchos contextos educativos hasta inicios del 2020, el desarrollo educativo de los estudiantes estaba determinado en gran medida por las actividades que tenían lugar en escuelas, institutos o universidades. Mientras que, en el presente, aunque también desde hace muchos años, Internet ofrece grandes oportunidades para que los estudiantes regulen su propio aprendizaje. Tienen las mejores bibliotecas, hemerotecas, exposiciones y foros de discusión a su alcance, sin restricciones de tiempo. No obstante, el acceso a tales recursos sigue estando ausente en aquellos lugares donde aún no llegan las conexiones Wifi.

La renovación y ampliación de los límites del aula, como contexto único de enseñanza y de aprendizaje, moviliza nuevas interacciones y evoca una mayor autonomía en los estudiantes, quienes desde sus intereses, motivaciones y metas formuladas movilizan recursos para modificar los escenarios educativos. Esto es, convoca a la agencia transformadora, definida como el potencial de los seres humanos para convertirse en creadores de historias; o transformadores de sus propias actividades, a partir de acciones específicas que tienen el potencial de transformación (Lemos, Pereira-Querol y Muniz de Almeida, 2013; Engeström, 2017).

Esta agencia, caracterizada como trasformadora, es la base para pensar en el aprendizaje expansivo. En un contexto particular de aprendizajes virtuales, atravesado por la pandemia covid-19, tiene un amplio alcance para pensar el retorno a las aulas. El desafío será generar una vez más redes entre agentes y comunidades, salir de la clase para expandir el clima del aula y potenciar el compromiso de los estudiantes. Ese reto no se limita a lo virtual. La sociedad, las comunidades de práctica y otras instituciones tienen mucho que aportar al aprendizaje de los estudiantes (Rigo y Guarido, 2020).

En el aprendizaje expansivo, Engeström y Sannino (2010) postulan que los estudiantes aprenden algo que aún no existe. Los alumnos construyen un nuevo objeto o concepto en el marco de sus actividades colectivas e implementan ese aprendizaje en la práctica. La formación de un objeto expandido y el nuevo patrón de comportamiento correspondiente generan una agencia distribuida transformadora, que cuestiona y rompe con las limitaciones de las actividades existentes, emprendiendo un viaje por tierras inexploradas de la zona de desarrollo próximo. Engeström (2000) define a la zona de desarrollo próximo como el espacio para la transición expansiva de las acciones a la actividad.

Se trata de un proceso cíclico, integrado por las siguientes acciones: cuestionamiento -de algunos aspectos de la práctica aceptada-, análisis - de la situación, rastreando orígenes y evolución-, modelado de una nueva solución -construir un modelo explicativo de la nueva idea que ofrezca una solución a la situación problemática-, examen y puesta a prueba del nuevo modelo - para comprender su dinámica, potenciales y limitaciones-, deliberación sobre el proceso y fortalecimiento y generalización de la nueva práctica - en una nueva modalidad estable- (Engeström y Sannino, 2016). 
El aprendizaje expansivo implica en su definición la agencia transformadora, generada en el proceso de la doble estimulación, el cual consiste:

En situaciones de incertidumbre y de incongruencia cognitiva como el experimento de espera, las personas se enfrentan a un conflicto motivacional. Gestionan dichos conflictos apoyándose en artefactos que cumplen la función de motivos auxiliares. Estos artefactos, o estímulos secundarios, les ayudan a realizar acciones volitivas y transformar la situación. Esta interpretación de la doble estimulación está basada en un texto de Vygotsky (1997) sobre el autocontrol. (Engeström y Sannino, 2016, p. 420)

Es decir, en la doble estimulación, Vygotsky (1997) ofrece al sujeto no solo una tarea a resolver (primer estímulo), sino que además le brinda un artefacto externo ambiguo (segundo estímulo), al cual los sujetos le otorgan significado, hasta convertirse en un nuevo signo mediador, para mejorar sus acciones y conducir una reformulación de la tarea. El aprendizaje expansivo, generalmente, requiere de intervenciones formativas basadas en el principio de doble estimulación.

Las intervenciones formativas son definidas como "una metodología que intenta superar las dificultades para trasladar al campo de las prácticas pedagógicas los resultados y avances logrados por la investigación educativa" (Rinaudo, 2007, p. 75). Sannino, Engeström y Lemos (2016) puntualizan que, basados en la teoría de la actividad, pueden generar un nuevo tipo de diálogo y complementariedad entre el impacto práctico y el análisis riguroso; metodología que genera una transformación cualitativa de los procesos de aprendizaje, un cambio comprendido como un proceso largo y distribuido, a través de la organización social y reflexiva, la cual se concretiza en la creación de redes de aprendizaje que trascienden los límites institucionales de la escuela, redefinida como un instrumento colectivo (Yamazumi, 2009).

Erausquin (2014) sintetiza que el aprendizaje expansivo se caracteriza por ser transformador, al ampliar los objetos compartidos en el sistema de actividad por medio de herramientas y recursos nuevos; experiencial, al colocar a los agentes en situaciones simuladas y reales, que requieren de compromiso personal en acciones con objetos, que incluyen a otros, anticipando un nuevo modelo de comportamiento; horizontal y dialógico, en tanto se transforma la actividad a partir de la generación de nuevos conocimientos, siguiendo la lógica de contextos híbridos, fundando lazos entre sistemas de actividad diversos; y, por último, subterráneo, al desarrollar nuevos métodos que, sin ser notados, se cuelan entre los intersticios de las estructuras antiguas y estables que sostienen su viabilidad.

Yamazumu (2008) agrega que, bajo esas características, el aprendizaje expansivo, en términos de la tercera generación de la teoría de actividad, supera los límites de un único sistema y adopta como unidad de análisis múltiples sistemas de actividad que interactúan mutuamente, promoviendo la intervención empírica para diseñar e implementar redes, diálogos y colaboraciones entre los sistemas.

El desafío es transformar el sistema educativo, expandiendo el objeto de aprendizaje, al crear puentes entre múltiples contextos, promover la ruptura del aprendizaje encapsulado y eventualmente la formación y creación de la autoorganización colaborativa y redes que trascienden los límites de las instituciones educativas. En términos de compromiso académico, supone abrir el aula y generar aprendizajes interconectados con la comunidad para que el conocimiento ligado a una utilidad genuina se transforme y posibilite la toma de decisiones de manera crítica.

Aprender expansivamente requiere romper con el marco de acción, dado que si miramos los contextos educativos actuales, comprometen poco a los estudiantes; también precisa tomar la iniciativa de transformarlos en pos de prácticas que propicien la implicación académica; tarea en la cual el vínculo con las TIC y las comunidades locales es imprescindible (Rigo et al., 2020). Al respecto, Sannino et al. (2016) exponen que las nuevas prácticas generadas en un proceso de aprendizaje expansivo se vinculan con las iniciativas y los compromisos de los alumnos. Hacia ese lugar la educación se debe proyectar. 


\section{Consideraciones finales}

El presente artículo se propuso desarrollar una revisión teórica de los principales aportes sobre agencia desde la teoría de la actividad, en tanto cobra relevancia en los últimos planteos sobre compromiso. Dimensión proactiva, propuesta como cuarta faceta de un metaconstructo con manifestaciones afectivas, conductuales y cognitivas, a la vez maleable, situado y distribuido.

La revisión, en el marco de la agenda educativa formulada por la UNESCO al 2030, es elocuente para trazar metas a futuro. Una educación que tienda "Garantizar una educación inclusiva y equitativa de calidad y promover oportunidades de aprendizaje permanente para todos" (UNESCO, 2016, p. 7). La Educación 2030 debe encuadrarse en un contexto más amplio, sin desconocer los avances tecnológicos, de urbanización, de los mercados laborales, los desastres medioambientales, entre un sinfín de problemáticas que atraviesan las sociedades. Pensarlas como herramientas para facilitar el diálogo y promover el respeto por la diversidad.

Hacemos propia la meta de alcanzar una educación que tenga reparos en la sociedad, que no se aísle, que forme a sujetos reflexivos y metacognitivos. Asumimos en el rol de la agencia un eslabón clave para lograrla, atendiendo a las particularidades de cada contexto y cada sujeto, con propuestas de enseñanza y de aprendizaje que tiendan a generar vínculos entre instituciones educativas y sociales. Multiplicar las interrelaciones entre los sistemas de actividad, construir objetos nuevos a partir de una agencia múltiple, distribuida y en red que busque andamiar respuestas situadas en pos de una educación expandida hacia la sociedad, que comprometa a los estudiantes universitarios, como sujetos activos en la construcción del conocimiento.

En el reto de generar vínculos variados y diversos entre sistemas encontramos la riqueza de la revisión realizada, a fin de atender a futuras intervenciones formativas de cara a generar rupturas en los sistemas educativos encapsulados, en vista a caminar por nuevas zonas de desarrollo que permitan el paso de las acciones a las actividades (Engeström, 2000).

\section{Referencias}

Arrúe, C. y Elichiry, N. (2014). Aprendizaje situado, actividad e interactividad. Análisis de talleres de juego en la escuela primaria. Anuario de Investigaciones, XXI, 65-73. http://catalogosuba.sisbi.uba. ar/vufind/Record/KOHA-OAI-APS:48985

Ávila Reyes, N., Navarro, F. y Tapia-Ladino, M. (2020). Identidad, voz y agencia: claves para una enseñanza inclusiva de la escritura en la universidad. Archivos Analiticos de políticas Educativas,28(98), 1-27. https://doi.org/10.14507/ epaa.28.4722

Bandura, A. (1989). Human Agency in Social Cognitive Theory. American Psychologist, 44(9), 1175 1184. http://www.uky.edu/ - eushe2/Bandura/ Bandura1989AP.pdf

Bandura, A. (1999). Social cognitive theory of personality. In L. A. Pervin \& O. P. John (Eds.), Handbook of personality: Theory and research (pp. 154-196). Guilford Press

Bandura, A. (2001). Social Cognitive Theory: An Agentic Perspective. Annu. Rev. Psychol., 52, 1-26. www.annualreviews.org

Bandura, A. (2006). Toward a psychology of human agency. Perspectives on Psychological Science, 1, 164-180.https://doi.org/10.1111/j.1745-6916. 2006.00011.x

Bourdieu, P. (1997). El sentido práctico. Siglo XXI Editores.

Engeström Y. (2017). Expanding the Scope of Science Education: An Activity-Theoretical Perspective. In Hahl K., Juuti K., Lampiselkä J., Uitto A., Lavonen J. (eds), Cognitive and Affective Aspects in Science Education Research. Contributions from Science Education Research, vol 3. Springer, Cham. https://doi.org/10.1007/9783-319-58685-4_26

Engeström, Y. (1991). Non scolae sed vitae discimus: Toward overcoming the encapsulation of school learning. Learning and Instruction, I, 243-259. https://doi.org/10.1016/0959-4752 (91)90006-T 
Engeström, Y. (2000). From individual action to collective activity and back: Developmental work research as an interventionist methodology. In P. Luff, J. Hindmarsh, y C. Heath (Eds.), Workplace studies. Cambridge University Press.

Engeström, Y. (2001). Expansive Learning at Work: Toward an activity theoretical reconceptualization. Journal of Education and Work, 14(1), 133-155. https://doi.org/10.1080/136390800 20028747

Engeström, Y. (8-13 de septiembre de 2008). The future of activity theory: a rough draft. [Conferencia magistral]. ISCAR Congress, WorldWide Conference on Role of Culture in Human Development, San Diego, Estados Unidos. http://lchc.ucsd.edu/MCA/Paper/ISCARkeyEngestrom.pdf

Engeström, Y. \& Sannino, A. (2010). Studies of expansive learning: Foundations, findings and future challenges. Educational Research Review, 5(1), 1-24. https://doi.org/10.1016/j.edurev. 2009.12.002

Engeström, Y. \& Sannino, A. (2016). Expansive learning on the move: Insights from ongoing research / El aprendizaje expansivo en movimiento: aportaciones de la investigación en curso. Infancia y Aprendizaje, 39(3), 401-435. https://doi.org/10. 1080/02103702.2016.1189119

Erausquin, C. (2014). La Teoría Histórico-Cultural de la Actividad como artefacto mediador para construir Intervenciones e Indagaciones sobre el Trabajo de Psicólogos en Escenarios Educativos. Revista Segunda Epoca, 13, 173-197. https:// revistas.unlp.edu.ar/revpsi/article/view/1116

Gutiérrez, K., Engeström, Y. \& Sannino, A. (2016). Expanding Educational Research and Interventionist Methodologies. Cognition and Instruction, 34(3), 275-284. http://dx.doi.org/10.108 0/07370008.2016.1183347

Kuckartz, U. \& Rädiker, S. (2019). Analyzing Qualitative Data with MAXQDA. Springer.

Laitinen, A., Sannino, A. \& Engeström, Y. (2016). Rom controlled experiments to formative inter- ventions in studies of agency: Methodological considerations. Educação, 39, s14-s23. http:// revistaseletronicas.pucrs.br/ojs/index.php/ faced/article/view/24321

Larreamendy Joerns, J (2011). Aprendizaje como reconfiguración de agencia. Revista de Estudios Sociales, 40, 33-43. http://www.redalyc.org/ articulo.oa? $\mathrm{id}=81522330004$

Lemos, M., Pereira-Querol, M.A.y Muniz de Almeida, I. (2013). A Teoria da Atividade Histórico-Cultural e suas contribuiçôes à Educação, Saúde e Comunicação: entrevista com Yrjö Engeström. Interface - Comunicação, Saúde, Educação, 17(46), 715-727. https://doi.org/10.1590/ S1414-32832013000300018

Leóntiev, A.N. (1989). Actividad, conciencia, personalidad. En A. Puziréi (comp.), El proceso de formación de la psicología marxista: L. Vygotski, A. Leóntiev, A. Luria (pp. 265-326). Progreso.

Montenegro, A. (2017). Understanding the Concept of Agentic Engagement for Learning. Colomb. Appl. Linguist. J., 19(1), 117-128. http://dx.doi. org/10.14483/calj.v19n1.10472

Reeve, J. (2013). How Students Create Motivationally Supportive Learning Environments for Themselves: The Concept of Agentic Engagement. Journal of Educational Psychology, 105(3), 579-595. https://bmri.korea.ac.kr/file/board_ data/publications/1414975413_1.pdf

Reeve, J. \& Shin, S. (2020). How teachers can support students' agentic engagement. Theory Into Practice, 59(2), 150-161. https://doi.org/10.1080/0 0405841.2019 .1702451

Reeve, J. \& Tseng, C-M. (2011). Agency as a fourth aspect of students' engagement during learning activities. Contemporary Educational Psychology, 36, 257-267. https://doi.org/10.1016/j. cedpsych.2011.05.002

Reeve, J. \& Jang, H. (2006). What teachers say and do to support students' autonomy during a learning activity. Journal of Educational Psychology, 98, 209-218. https://eric.ed.gov/?id= EJ734398 
Rigo, D. (en prensa) (Comp.). Compromiso y educación. Redefinición del aula como contexto de aprendizaje. Colección Mundo Digital de Revista Mediterránea de Comunicación. Universidad de Alicante.

Rigo, D. y Donolo, D. (2018). ¿Es posible invertir la forma en que aprendemos y enseńamos? Aderezos para repensar la educación. Revista Innovaciones Educativas, $X X(28)$, 106-119. http://investiga. uned.ac.cr/revistas/index.php/innovaciones/ article/view/2135/2501

Rigo, D. y Donolo, D. (2019). Análisis de un modelo integrador del compromiso escolar: relaciones entre variables situacionales, escolares, sociales y personales en alumnos de nivel primario de educación. Propósitos y Representaciones, 7(SPE), e316. http://dx.doi.org/10.20511/pyr2019. v7nSPE.316

Rigo, D. y Guarido, G. (2020). Mirar la clase universitaria. Compromiso académico, vínculo con el clima de aula. En R. Rivera Espinosa (Coord.), $V$ Congreso online Internacional sobre la Educación en el Siglo XXI (pp.130-143). Servicios Académicos Intercontinentales, EUMED. https://www.eumed.net/actas/20/educacion/ educacion20.pdf

Rigo, D., Irusta, M., Bechero, G. y Amaya, S. (2020). Motivos para comprometerse, desvincularse y revincularse con los estudios superiores. Investigación en la Escuela, 100, 71-87. https://dx.doi. org/10.12795/IE.2020.i100.06

Rinaudo, M. C. (2007) Investigación educativa. Ideas para pensar la formación de investigadores. En Donolo, D., y Rinaudo, M. C. (2007), Investigación en educación: aportes para construir una comunidad más fecunda (pp. 55-84). La Colmena.

Rogoff, B. (1993). Aprendices del Pensamiento. El desarrollo cognitivo en el contexto social. Paidós.

Rogoff, B. (1997). Los tres planos de la actividad sociocultural. En Wertsch, J., Del Rio, P. y Álvarez, A. (Eds), La mente sociocultural, aproximaciones teóricas aplicadas (pp. 11-128). Infancia y aprendizaje.

Sannino, A., Engeström, Y. \& Lemos, M. (2016). Formative interventions for expansive learning and transformative agency. Journal of the Learning Sciences, 25(4), 599-633. https://doi.org/ 10.1080/10508406.2016.1204547

Sautu, R. (2014). Agencia y estructura en la reproducción y cambio de las clases sociales. Theomai, 29, 100-120. http://www.redalyc.org/articulo. oa? $\mathrm{id}=12431432006$

UNESCO. (2016). Educación 2030. Declaración de Incheon. Hacia una educación inclusiva y equitativa de calidad y un aprendizaje a lo largo de la vida para todos. http://www.unesco.org/new/fileadmin/MULTIMEDIA/FIELD/Santiago/pdf/ ESP-Marco-de-Accion-E2030-aprobado.pdf

Vygotski, L.S. (1993). Pensamiento y lenguaje. En L. S. Vygotski, Obras escogidas, II. Aprendizaje Visor.

Vygotsky, L. S. (1997). The history of the development of higher mental functions. In R. W. Rieber (Ed.), The collected works of L. S. Vygotsky. Vol. 4: The history of the development of higher mental functions. Plenum.

Wertsch, J. (1998). La mente en acción. Aique.

Yamazumi, K. (2006). Activity Theory and the Transformation of Pedagogic Practice. Educational Studies in Japan: International Yearbook, 1, 77-90. https://ci.nii.ac.jp/naid/110006244668

Yamazumi, K. (2007). Human Agency and Educational Research: A New Problem in Activity Theory. Actio: An International Journal of Human Activity Theory, 1, 19-39. https://core. ac.uk/display/59116674

Yamazumi, K. (2009). Expansive Agency in Multi-Activity Collaboration. En Sannino, A., Daniels, H. \& Gutiérrez, K. (ed.), Learning and Expanding with Activity Theory (pp. 212-227). Cambridge University Press.

Yamazumu, K. (2008). A hybrid activity system as educational innovation. J Educ Change, 9, 365-373. https://link.springer.com/article/ z10. 1007/s10833-008-9084-8

Zuckerman, G. (2007). On supporting children's initiative. Journal of Russian and East European Psychology, 45(3), 9-42. https://psyjournals.ru/ en/kip/2007/n1/Tsukerman.shtml 